\title{
Racial/Ethnic Differences in Labor Outcomes with Prostaglandin Vaginal Inserts
}

\author{
Megan L. Stephenson • J. Seth Hawkins • Leo Pevzner • \\ Barbara L. Powers • Deborah A. Wing
}

Received: 19 February 2014 /Revised: 26 August 2014 / Accepted: 4 September 2014 /Published online: 2 October 2014

(C) W. Montague Cobb-NMA Health Institute 2014

\begin{abstract}
Objective The aim of this study is to compare labor outcomes across race/ethnicity in women undergoing prostaglandin labor induction.

Methods Secondary analysis of misoprostol vaginal insert (MVI) trial, a double-blind, randomized, control trial of 1,308 patients comparing sustained release vaginal inserts containing dinoprostone $10 \mathrm{mg}$ and misoprostol $50 \mathrm{mcg}$ (MVI 50) or $100 \mathrm{mcg}$ (MVI 100).

Results Achievement of active labor and induction failures were similar across race/ethnicity. Cesareans were performed less frequently in whites (29\%) and Hispanics (24.5\%) compared to blacks $(32.7 \%)$ (adjusted odds ratio (aOR) $0.87,95 \%$ confidence interval (CI) $0.47-0.97, p=$ 0.03 and aOR $0.86,95 \%$ CI $0.44-0.97, p=0.03$, respectively). When compared to blacks, whites were less likely
\end{abstract}

M. L. Stephenson $(\bowtie) \cdot$ D. A. Wing

Division of Maternal-Fetal Medicine, Department of Obstetrics and Gynecology, University of California Irvine Medical Center, Orange, CA 92868, USA

e-mail: meganls@uci.edu

J. S. Hawkins

Division of Maternal-Fetal Medicine, Department of Obstetrics \& Gynecology, University of Texas Southwestern Medical Center at Dallas, Dallas, TX 75235, USA

L. Pevzner

Department of Obstetrics and Gynecology, Kaiser Permanente Orange County, Irvine, CA 92618, USA

\section{B. L. Powers}

Independent Consultant, Phoenixville, PA 19460, USA

M. L. Stephenson • D. A. Wing

Department of Obstetrics and Gynecology, Memorial Care Center for Women at Miller Children's Hospital, Long Beach Memorial Medical Center, Long Beach, CA 90806, USA to undergo cesarean for non-reassuring fetal heart rate tracing (aOR 0.41, $95 \%$ CI 0.25-0.66, $p=0.0003$ ), as were Hispanics (aOR 0.38, $95 \%$ CI 0.22-0.65, $p=0.0004)$. Postpartum hemorrhage occurred more frequently in Hispanics $(8.8 \%)$ versus blacks (4.1\%) and whites (OR 2.27, $95 \%$ CI 0.23-0.82, $p=0.02$ and OR 3.69 , $95 \%$ CI $0.14-0.51, p<0.0001$, respectively). Birth weights of black infants were lower than whites $(p<0.0001)$ and Hispanics $(p=0.0003)$. Neonatal outcomes did not differ between groups.

Conclusion Differences in labor induction outcomes with prostaglandin labor induction exist based on race/ethnicity. Blacks delivered smaller babies, were more likely to undergo cesarean, and have cesareans performed for non-reassuring fetal heart tracing compared to other groups. Hispanics were more likely to experience postpartum hemorrhage compared to the other races.

Keywords Disparity $\cdot$ Labor induction $\cdot$ Cesarean $\cdot$ Race

\section{Introduction}

Significant racial and ethnic disparities exist throughout health care and are particularly evident in obstetrics where differences have been well established in all aspects of pregnancy care. These disparities have persisted over time and profoundly impact maternal and infant outcomes, future pregnancy care, and health care costs. Many studies have sought to clarify the causes of these disparities by examining socioeconomic status, environmental exposures, genetic predisposition, maternal stress, infection, and differences in provision of health care [2, 6-8]. However, accurately measuring outcomes and identifying quality indicators with respect to racial/ ethnic differences have been difficult due to the varying definitions of disparities used throughout the literature and 
the vast amount of potential confounding factors [7]. This is reflected in the relatively few standardized measures of quality obstetric care, such as time of entry into prenatal care, and has prompted investigation into other potential quality indicators such as cesarean delivery rate, postpartum hemorrhage and puerperal infection $[2,7,9]$. Just as a woman who entered spontaneous labor has a different risk profile than one who underwent an induction, these outcomes are intimately linked to a woman's health status at the time of labor and what occurred intrapartum.

This secondary analysis sought to address differences in intrapartum variables and labor outcomes based on race/ ethnicity in women undergoing induction of labor with misoprostol or dinoprostone vaginal inserts and suggests possible causes. Vital to all investigation into racial disparities is the goal of revealing modifiable differences above and beyond those that can be explained by differences in health status between groups. This study focused mainly on intrapartum and outcome variables in an attempt to identify if there was a more immediate temporal relationship between any of the disparities identified or if the modifiable aspects were based on prelabor factors such as prenatal exposures, genetic predisposition, and social or behavioral factors. In this population of women undergoing induction of labor with prostaglandins, we hypothesized that women of color would have disproportionately poorer outcomes when compared to white women including higher cesarean rates, more postpartum hemorrhage, and greater rates of puerperal infection after controlling for possible confounders. We also hypothesized that some historically persistent disparities would again be noted such as smaller babies born to black mothers.

\section{Methods}

The study was a secondary analysis of the data collected from the misoprostol vaginal insert (MVI) randomized control trial [10]. The original trial, Miso-Obs-004, was a multisite, double-blind, randomized study comparing the efficacy and safety of three different sustained release removable vaginal inserts containing dinoprostone $10 \mathrm{mg}$, misoprostol $50 \mathrm{mcg}$ (MVI 50), or misoprostol $100 \mathrm{mcg}$ (MVI 100). Forty-nine institutions across 24 states and 3 Canadian provinces participated in the trial, and 1,308 patients were enrolled from April 26, 2006 to August 7, 2007. The Institutional Review Board at the University of California Irvine approved the study.

Enrollment criteria, induction indication definitions, and a more detailed description of the methods for this study can be found in the original publication of the MVI trial [10]. In brief, participants in the original study were recruited locally at each site from investigators' private practices, referrals from other attending physicians, and obstetric clinics. The participants were aged at least 18 years, of low parity ( 3 or less) with singleton pregnancies, and at least 36 weeks 0 days of gestation. They had no conditions requiring urgent delivery and required cervical ripening defined as baseline modified Bishop score of 4 or less. The admitting physician recorded the indication for induction. Participants were excluded if labor or vaginal delivery was contraindicated, delivery was deemed to be urgent, or sensitivity to either misoprostol or dinoprostone was known.

The original MVI trial presented the data on 1,308 patients using an intent-to-treat analysis. Wing et al. concluded that the median time intervals to vaginal delivery for both the MVI $100 \mathrm{mcg}$ and the dinoprostone $10 \mathrm{mg}$ vaginal insert were similar, whereas the MVI $50 \mathrm{mcg}$ had a significantly longer time to vaginal delivery [10]. The three removable inserts were also found to have similar safety profiles as well as cesarean rates. The current analysis was confined to the 1,274 patients with sufficient outcome data who completed the study and delivered during their first admission. The study sponsor (Controlled Therapeutics (Scotland) Ltd.) used all available data collected in the course of the trial to construct a comprehensive database that was made available to the current authors for secondary analyses.

Maternal race/ethnicity was recorded as stated by the patient upon admission as white, black, Hispanic, Asian, Pacific Islander, or others. In all, there were 587 patients who identified themselves as white, 245 as black, 363 as Hispanic, 32 as Asian, and 47 as others. Given the small number of participants classified as Asian, Pacific Islander, and others, meaningful comparison could not be undertaken, and as such, these races were excluded from this secondary analysis.

Following study enrollment, an examination was performed to confirm vertex fetal presentation and to determine baseline modified Bishop score. Modified Bishop score was calculated again at $12 \mathrm{~h}$ after study drug insertion. Time to delivery was determined as the interval from insertion of the study drug to delivery of the fetus. Primary reason for discontinuation of the study drug insert included onset of active labor, study drug falling out of the vagina, completion of the 24-h dosing period and/or maternal-fetal complications. Removal of study drug after $24 \mathrm{~h}$ without evidence of active labor was categorized as "failed induction" in final analysis. Active labor was defined as at least three firm, rhythmic contractions lasting at least $45 \mathrm{~s}$ within a $10-\mathrm{min}$ period or achievement of at least 4-cm dilatation. Frequency, duration, and amount of predelivery oxytocin use were recorded. Adverse events were recorded for both the mother and her neonate. These included a variety of outcomes such as postpartum hemorrhage, uterine contractile abnormalities, and side effects to the medication (maternal), as well as such neonatal outcomes as admission to the neonatal intensive care unit (NICU), hypoglycemia and hyperbilirubinemia. Differences between the treatment arms based on race were 
not examined as the subject number in each of the groups did not allow for meaningful comparison.

Statistical analyses were performed using JMP 9.0 (SAS Institute Inc., Cary, NC) statistical software, and all tests were conducted at the 0.05 significance level. Race/ethnicity was the basis of this comparison. Continuous variables were evaluated using Student's $t$ tests where appropriate for normally distributed data or Wilcoxon rank-sum test for nonparametric data. Means were compared between multiple race/ethnicity pairs using ANOVA and Tukey-Kramer HSD method. Categorical variables were evaluated using $\chi^{2}$ test, Fisher's exact test, Mann-Whitney $U$, or other tests as appropriate.

\section{Results}

Data from 1,274 patients was analyzed, of which $46 \%$ identified themselves as white, $28 \%$ as Hispanic, $19 \%$ as black, and $6 \%$ as others, Asian, or Pacific Islander. As stated above, the Asians, Pacific Islanders and participants describing themselves as other were excluded, thus 1,195 women were included. There was normal distribution of the races among the different treatment groups. Table 1 describes participant characteristics.
As shown in Table 1, maternal age varied significantly by race, with black participants being of the lowest mean age (24.9 \pm 5 .6; Hispanic $25.6 \pm 5.7$, and whites $27.1 \pm 5.9$ ). The mean age for whites was significantly older than Hispanics $(p=0.002)$ and blacks $(p=<0.0001)$; however, there was no significant difference between Hispanics and blacks $(p=0.28)$. Body mass index (BMI) was calculated using maternal weight at time of admission for induction. Prepregnancy BMI and total weight gain in pregnancy were not available from all participants so was not included in the analysis. Black women had a higher mean BMI than whites or Hispanics though this was only significant when blacks were compared to whites $(p=$ $<0.0001)$. There was a higher proportion of white women in the BMI $<30$ group $(38.8 \%)$ compared to blacks $(25.3 \%)$, and there were more Hispanic women in the BMI 30-40 group compared to other races/ethnicities. The largest proportion of the BMI $>40$ group were black women (22.4\%). Nulliparity was encountered more often in white women $(66.3 \%)$ than Hispanics $(57.6 \%)$ or blacks $(57.6 \%),(p=0.008)$.

Significant differences $(p<0.0001)$ between the races were noted when reason for induction was analyzed, with elective being the most common, with $56.4 \%$ of whites, $48.6 \%$ of blacks, and $44.4 \%$ of Hispanics induced electively. Hypertensive disorders of pregnancy (hypertension and preeclampsia) as a group were the next most common reasons for

Table 1 Demographic and baseline characteristics based on race/ethnicity

\begin{tabular}{|c|c|c|c|c|c|c|}
\hline Characteristics & White $(n=587)$ & Hispanic $(n=363)$ & Black $(n=245)$ & $p$ value $^{\mathrm{a}}$ & $p$ value $^{\mathrm{b}}$ & $p$ value ${ }^{\mathrm{c}}$ \\
\hline Maternal age (year) & $27.1 \pm 5.9$ & $25.6 \pm 5.7$ & $24.9 \pm 5.6$ & 0.002 & $<0.0001$ & 0.28 \\
\hline Mean BMI & $33.0 \pm 6.6$ & $34.1 \pm 6.5$ & $35.4 \pm 8.0$ & 0.053 & $<0.0001$ & 0.06 \\
\hline $\mathrm{BMI}<30$ & $228(38.8)$ & $100(27.6)$ & $62(25.3)$ & & & \\
\hline BMI 30-40 & $272(46.3)$ & $207(57.2)$ & $128(52.2)$ & & & \\
\hline $\mathrm{BMI} \geq 40$ & $87(14.8)$ & $55(15.2)$ & $55(22.4)$ & & & \\
\hline Parity & & & & 0.008 & & \\
\hline Nulliparous & $389(66.3)$ & $209(57.6)$ & $141(57.6)$ & & & \\
\hline Parous & $198(33.7)$ & $154(42.4)$ & $104(42.4)$ & & & \\
\hline Gestational Age (weeks) & $39.5 \pm 1.4$ & $39.8 \pm 1.4$ & $39.5 \pm 1.3$ & 0.003 & 0.96 & 0.045 \\
\hline Baseline Bishop score & $2.84 \pm 1.04$ & $2.67 \pm 1.10$ & $2.55 \pm 1.17$ & 0.06 & 0.002 & 0.40 \\
\hline Reason for induction & & & & $<0.0001$ & & \\
\hline Elective & $331(56.4)$ & $161(44.4)$ & $119(48.6)$ & & & \\
\hline Hypertension & $77(13.1)$ & $27(7.4)$ & $30(12.2)$ & & & \\
\hline Preeclampsia & $49(8.4)$ & $24(6.6)$ & $19(7.8)$ & & & \\
\hline Diabetes & $30(5.1)$ & $48(13.2)$ & $21(8.6)$ & & & \\
\hline Ruptured membranes & $16(2.7)$ & $25(6.9)$ & $10(4.1)$ & & & \\
\hline Oligohydramnios & $46(7.8)$ & $51(14.1)$ & $26(10.6)$ & & & \\
\hline Cholestasis & $3(0.5)$ & $6(1.6)$ & $1(0.4)$ & & & \\
\hline$\geq 42$ weeks & $4(0.7)$ & $5(1.4)$ & $3(1.2)$ & & & \\
\hline Others & $31(5.3)$ & $16(4.4)$ & $16(6.5)$ & & & \\
\hline
\end{tabular}

${ }^{\text {a }}$ White versus Hispanic

${ }^{\mathrm{b}}$ White versus Black

${ }^{\mathrm{c}}$ Hispanic versus Black 
induction, although there were proportionately fewer Hispanics induced for either hypertension or preeclampsia compared to blacks and whites, with relatively more Hispanics induced for diabetes and oligohydramnios. Gestational age at delivery differed among the races with Hispanics delivering at $39.8 \pm 1.4$ weeks compared to whites at $39.5 \pm 1.4$ weeks $(p=0.003)$ and blacks at $39.5 \pm 1.3$ days $(p=0.045)$. There was no significant difference in gestational age between whites and blacks $(p=0.96)$. Baseline modified Bishop score was also found to differ by race/ethnicity, with blacks showing a significantly lower Bishop score at baseline compared to whites $(p=0.002)$.

Nonetheless, this significant difference in baseline modified Bishop score did not affect the odds of reaching active labor, as seen in Table 2, and this analysis was not affected by adjusting for age, race, parity, BMI, gestational age at admission, and baseline modified Bishop score (data not shown). Additionally, time in active labor and time to delivery did not differ significantly by race/ethnicity (see Table 3 ). However, as seen in Table 2, black women were less likely than white women to require oxytocin and were more likely to deliver by cesarean compared to Hispanic women. After adjusting for age, race, parity, BMI, gestational age at admission, and baseline modified Bishop score, the lower odds of requiring oxytocin for blacks relative to whites (adjusted odds ratio (aOR) 0.68, $95 \%$ confidence interval (CI) $0.46-0.94, p=0.02$ ) and the lower rate of cesarean among Hispanics compared to blacks (aOR $0.86,95 \%$ CI $0.44-0.97, p=0.03$ ) remained significant. Additionally, after such adjustments were made, whites were also less likely to deliver by cesarean relative to blacks (aOR 0.87, $95 \%$ CI 0.47-0.97, $p=$ 0.03 ). When compared to blacks, whites were less likely to undergo cesarean for non-reassuring fetal heart rate tracing (aOR 0.41, $95 \%$ CI $0.25-0.66, p=0.0003$ ), as were Hispanics (aOR 0.38, $95 \%$ CI $0.22-0.65, p=$ $0.0004)$. White women were less likely to deliver in $12 \mathrm{~h}$ versus the other races (Hispanics (OR 0.53, $95 \%$ CI $0.34-0.83, p=0.01$ ), and blacks (OR 0.44, $95 \%$ CI $0.27-0.71, p=0.01)$, but this significant difference was not noted at $24 \mathrm{~h}$. These findings for delivery within $12 \mathrm{~h}$ remained significant after adjustment for age, race, parity, BMI, gestational age at admission, and baseline modified Bishop score (whites versus Hispanics, aOR $0.54,95 \%$ CI $0.34-0.88, p=0.01$; whites versus blacks, aOR $0.40,95 \%$ CI $0.25-0.68, p=0.0007$ ). Chorioamnionitis and endometritis were diagnosed more often in Hispanic women than white women, and this difference remained after adjustment for age, race, parity, BMI, gestational age at admission, and baseline modified Bishop score for both chorioamnionitis (whites versus Hispanics aOR 0.34, $95 \%$ CI $0.18-0.62, p=0.0004$ ) and endometritis (whites versus Hispanics aOR $0.29,95 \%$ CI $0.09-0.88, p=0.03$ ) (Table 2). There was no difference in puerperal infection noted between black and Hispanic women. Additionally, Hispanics had a greater odds of postpartum hemorrhage compared to blacks (aOR 2.41, $95 \%$ CI 1.19-5.28, $p=0.01$ ) and compared to whites (aOR 3.69, $95 \%$ CI $0.14-0.51, p<0.0001$ ).

This greater risk of infection and postpartum hemorrhage did not significantly affect maternal length of stay (Table 3) nor were neonatal outcomes affected (Table 4). There were no significant differences between 5-min APGAR scores, the proportion of neonates with a 5-min APGAR less than 7, the proportion of neonates admitted to the intensive care unit (NICU), duration of stay in the NICU, or the proportion of neonates suffering any sort of adverse event. Black neonates were significantly lower in weight compared to white $(p=<0.0001)$ or Hispanic neonates $(p=0.0003)$.

Table 2 Intrapartum outcomes (categorical variables, unadjusted) based on race/ethnicity

\begin{tabular}{|c|c|c|c|c|c|c|c|c|c|}
\hline \multirow[t]{2}{*}{ Outcome } & \multirow{2}{*}{$\begin{array}{l}\text { White } \\
(n=587)\end{array}$} & \multirow{2}{*}{$\begin{array}{l}\text { Hispanic } \\
(n=363)\end{array}$} & \multirow{2}{*}{$\begin{array}{l}\text { Black } \\
(n=245)\end{array}$} & \multicolumn{2}{|c|}{ White versus Hispanic } & \multicolumn{2}{|l|}{ White versus black } & \multicolumn{2}{|c|}{ Hispanic versus black } \\
\hline & & & & OR $(95 \% \mathrm{CI})$ & $p$ value & OR $(95 \% \mathrm{CI})$ & $p$ value & OR $(95 \% \mathrm{CI})$ & $p$ value \\
\hline Reached active labor & $548(93.4)$ & $344(94.8)$ & $223(91)$ & $0.78(0.44-1.37)$ & 0.38 & $1.39(0.80-2.40)$ & 0.24 & $1.79(0.95-3.38)$ & 0.07 \\
\hline Oxytocin use & $449(76.5)$ & $270(74.4)$ & $171(69.8)$ & $1.1(0.83-1.52)$ & 0.46 & $1.4(1.01-1.96)$ & 0.046 & $1.26(0.88-1.80)$ & 0.21 \\
\hline Delivery $<12 \mathrm{~h}$ & $40(6.8)$ & $44(12.1)$ & $35(14.3)$ & $0.53(0.34-0.83)$ & 0.01 & $0.44(0.27-0.71)$ & 0.01 & $0.83(0.51-1.33)$ & 0.44 \\
\hline Delivery $<24 \mathrm{~h}$ & $282(48)$ & $177(48.76)$ & $124(50.6)$ & $0.97(0.75-1.26)$ & 0.83 & $0.9(0.67-1.22)$ & 0.50 & $0.93(0.67-1.28)$ & 0.65 \\
\hline Cesarean delivery & $170(29)$ & $89(24.5)$ & $80(32.7)$ & $1.25(0.93-1.69)$ & 0.14 & $0.84(0.61-1.16)$ & 0.29 & $0.67(0.47-0.96)$ & 0.03 \\
\hline NRFHT for cesarean & $54(31.8)$ & $26(29.2)$ & $39(48.8)$ & $1.13(0.65-1.97)$ & 0.78 & $0.49(0.28-0.84)$ & 0.01 & $0.43(0.23-0.82)$ & 0.01 \\
\hline Chorioamnionitis & $21(3.6)$ & $30(8.3)$ & $14(5.7)$ & $0.41(0.23-0.73)$ & 0.0024 & $0.61(0.31-1.22)$ & 0.16 & $1.49(0.77-2.87)$ & 0.23 \\
\hline Endometritis & $5(0.9)$ & $9(2.5)$ & $5(2)$ & $0.34(0.11-1.02)$ & 0.04 & $0.41(0.12-1.44)$ & 0.15 & $1.22(0.40-3.69)$ & 0.73 \\
\hline Postpartum hemorrhage & $15(2.6)$ & $32(8.8)$ & $10(4.1)$ & $0.27(0.14-0.51)$ & $<0.0001$ & $0.62(0.27-1.39)$ & 0.24 & $2.27(1.10-4.70)$ & 0.02 \\
\hline
\end{tabular}

Data presented as mean \pm standard deviation or $n(\%)$. 
Table 3 Intrapartum outcomes (continuous variables) based on race/ethnicity

\begin{tabular}{lllllll}
\hline Outcome & $\begin{array}{l}\text { White } \\
(n=587)\end{array}$ & $\begin{array}{l}\text { Hispanic } \\
(n=363)\end{array}$ & $\begin{array}{l}\text { Black } \\
(n=245)\end{array}$ & $\begin{array}{l}\text { White versus Hispanic } \\
p \text { value }\end{array}$ & $\begin{array}{l}\text { White versus black } \\
p \text { value }\end{array}$ & $\begin{array}{l}\text { Hispanic versus black } \\
p \text { value }\end{array}$ \\
\hline $\begin{array}{l}\text { Time in active labor (h) } \\
\quad \text { All subjects }\end{array}$ & $18.7 \pm 11.3$ & $19.3 \pm 11.1$ & $18.7 \pm 11.7$ & 0.46 & 0.96 & 0.59 \\
$\quad$ SVD only $(n=915)$ & $17.6 \pm 10$ & $18.4 \pm 10.8$ & $18.1 \pm 11$ & 0.34 & 0.88 & 0.78 \\
Time to delivery (hr) & & & & & 0.63 & 0.66 \\
$\quad$ All subjects & $26.9 \pm 12.7$ & $25.9 \pm 12.5$ & $26.4 \pm 13.8$ & 0.26 & 0.24 & 1.00 \\
$\quad$ SVD only ( $n=915)$ & $25.1 \pm 10.8$ & $24.2 \pm 11.9$ & $24.2 \pm 12.7$ & 0.33 & 0.79 & 0.89 \\
$\quad$ Length of stay-maternal (days) & $4.6 \pm 1.2$ & $4.6 \pm 1.3$ & $4.6 \pm 1.1$ & 0.91 & & \\
\hline
\end{tabular}

Data presented as mean \pm standard deviation or $n(\%)$

\section{Discussion}

We found significant differences in antepartum variables and intrapartum outcomes between the racial and ethnic groups included in this investigation of labor induction. Differences in intrapartum care-sensitive conditions including birth weight, rate of cesarean delivery, puerperal infection, and postpartum hemorrhage were noted and persisted after adjustment for age, race, parity, BMI, gestational age at admission, and baseline modified Bishop score.

White women included in this study tended to be older than the black and Hispanic women and were also more often nulliparous. Gestational age also differed with black and white mothers delivering at younger gestational ages than Hispanics. It is unclear if this is clinically significant as all of the participants were $\geq 36$ weeks and there were no differences noted in neonatal outcomes. The analysis was also not powered to look at more rare outcomes related to differences between late preterm and term infants. We also found that differences existed between the groups for indications for induction, such as a higher frequency of diabetes in Hispanics and hypertensive complications in both blacks and whites compared to other groups. It could be hypothesized that the lower gestational ages of blacks and whites at delivery could be related to this higher rate of hypertensive disorders for which earlier delivery is often indicated; however, the number of subjects in these groups is too small to come to any compelling conclusions in this regard. It should also be noted here that guidelines recommending against elective induction prior to 39 weeks had not yet been instituted at the time of this study [11-13]. Although the vast majority of patients that underwent elective induction did so after 39 weeks, this point is important to highlight. More white patients underwent elective induction versus blacks or Hispanics (56\% versus 44 and $49 \%$, respectively). It is unclear why this difference exists and some possible reasons explored in previous literature include physician bias, patient request, or regional differences in practice. Further exploration of these differences is warranted particularly in light of more current recommendations against elective delivery prior to 39 weeks [12].

Baseline Bishop score was also found to be statistically different though this is very unlikely to be clinically significant as all participants had a Bishop score of $<4$. Blacks had the lowest baseline Bishop score but significantly less black women received oxytocin. Some previous studies have attempted to establish a racial/ethnic difference in biochemical responses and pharmacologic variations based upon subtle molecular differences among race/ethnicities [14]. These include differences in molecular messaging pathways and receptor responses including those involved in differing responsiveness to prostaglandins such as dinoprostone and misoprostol. There has also been some exploration of differing responsiveness to prostaglandins in obese versus normal weight women [15-17]. Martinez et al. demonstrated that a higher BMI was associated with higher PGE2 levels in the rectal mucosa and postulated that PGE2 synthesis and response may be different in these patients [17]. Pevzner et al. also found that obese women who underwent induction with prostaglandins had a longer duration of labor, higher oxytocin requirement, and cesarean delivery rates [16]. The complete mechanism for these differences in obese women has not been characterized, and it could be hypothesized that there are differing rates of metabolism of certain prostaglandins or that there is some competitive inhibitor produced in these women that blunts the response to prostaglandins. It is also possible that there could be a selection bias in effect given that obesity disproportionally affects women of color. In this study, women with a BMI of 30-40 were more often Hispanic, and women with a BMI $>40$ were more often black. It is difficult to say whether the response to prostaglandins or to induction of labor itself is blunted by race, by BMI, or by a combination of the two. Most likely is that numerous factors affect the outcome, and these may not be able to ever be separated due to the inability to control for all variables. Although there has not been literature published on differing racial/ethnic response to prostaglandins in labor, there has been literature citing 


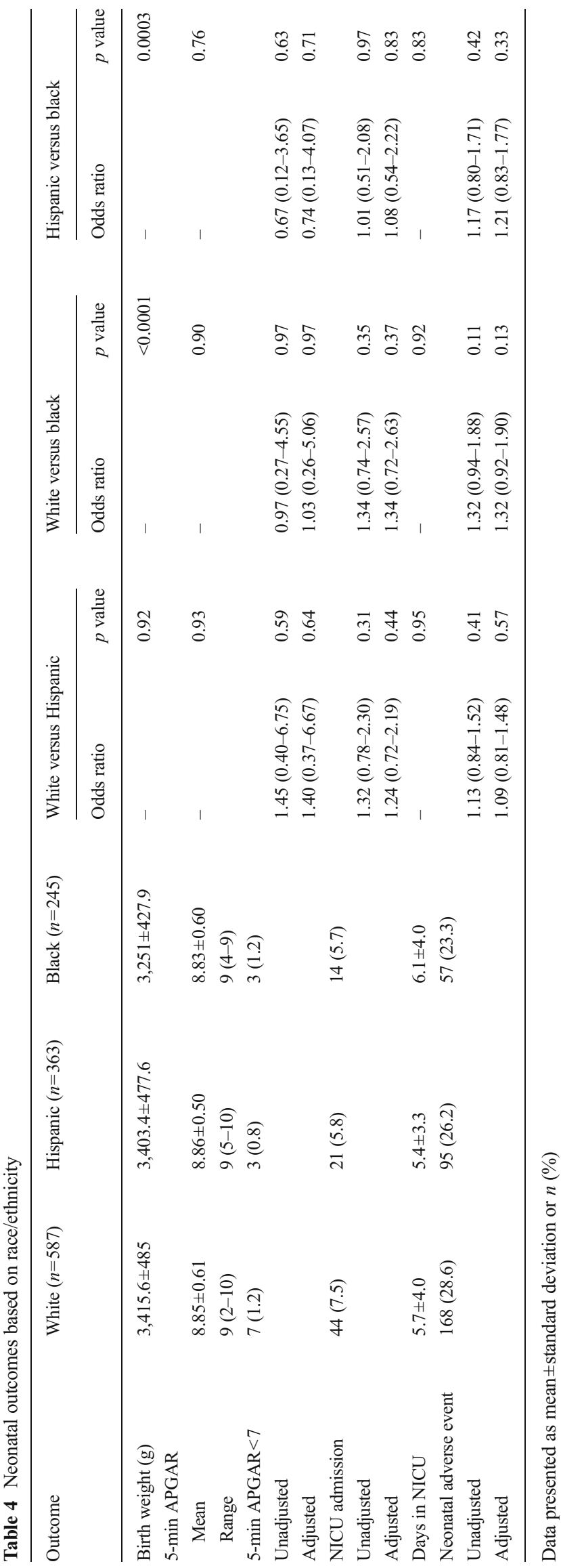

differing cytokine and inflammatory factor concentrations between races and the possible effects on preterm labor rates $[18,19]$. It is possible that genetic variation may be a more significant modifier on the ability to successfully and safely induce women. These studies present a possible avenue for further research on whether there is a basis for similar findings based on race/ethnicity.

Consistent with previous literature, black women in this study were found to have statistically smaller infants relative to other race/ethnicities $[3,4,7,8,20]$. Similar disparities in birth weight were described by Martin et al., who reported that blacks have a near 2-fold increase in low birth weight and a near 3-fold increase in very low birth weight infants compared to other race/ethnicities [20]. Birth weight is not affected by prostaglandin labor induction, so this variable was sought for a general comparison as this has been such an important measure of racial and ethnic disparity in the literature. Multiple factors have been cited as possible contributors to low birth weight in children of minority women including maternal health status prior to pregnancy and pregnancy weight gain, substance abuse, and exposure to certain environmental toxins have been associated with fetal growth restriction [8, 20, 21]. However, the effect of these exposures and health issues is not always consistent across races and even within certain racial/ethnic groups based upon their acculturation, country of birth, or spatial relationship to the USA [3, 22-24]. The CDC reported in 2002 that foreign born women had better outcomes than their US born racial/ethnic counterparts with regard to infant mortality, birth weight, and preterm birth rate despite later initiation of prenatal care and less education [3]. Interestingly, the difference in outcomes between foreign born and US born Latinas in particular has been shown to decrease with acculturation [25].

The majority of studies have demonstrated that higher cesarean rates exist among minority populations even after adjusting for known risk factors [1, 6, 26-29]. Chung et al. showed that compared with white patients, black patients were $75 \%$ and Hispanic patients were $22 \%$ more likely to undergo primary cesarean delivery [6]. Bryant et al. also observed a 1.48 and 1.19 times greater risk of cesarean delivery for African-Americans and Latina women respectively after adjustment for known risk factors [1]. After adjustment for age, race, parity, BMI, gestational age at admission, and baseline modified Bishop score, our results demonstrated a lower risk of cesarean delivery for both white women and Hispanic women compared to blacks but no significant difference between Hispanic and white women. In previous reports, Hispanics were often found to have lower cesarean rates when compared to other women of color from similar socioeconomic statuses, a phenomenon often referred to as the Hispanic paradox, which was supported by our findings [2, 23, 30]. Many researchers have attempted to identify the causes of this fairly consistent disparity though it appears to be related to 
multiple factors and may be further compounded by factors unable to be characterized or well measured.

The increasing prevalence of maternal obesity has influenced multiple areas of obstetric care and impacted both maternal and neonatal outcomes. The risk of cesarean delivery is known to be increased in obese women of all races and women that have greater than recommended weight gain during pregnancy [31-34] though not many studies have addressed the combined effect of both race and obesity on the risk of cesarean delivery $[31,35,36]$. The majority of reproductive age women in the USA are now overweight or obese with disproportionately more women of color affected. Marshall et al. in their evaluation of 312,412 women confirmed previous findings of increased risk of cesarean delivery in both obese Caucasian and obese African-Americans compared to their normal weight counterparts [31]. Interestingly though, they did note that there were no differences in the rate of cesarean delivery between the obese Caucasians compared to the obese African-Americans [31]. This was in contrast to findings by Steinfeld et al. in 2,424 women who found an increased cesarean rate in both obese Hispanic and AfricanAmerican women compared to obese white women [36]. Marshall et al. also found that there were persistent disparities among the neonates of obese mothers that had been demonstrated in infants of normal weight mothers. The neonates of the obese African-Americans were less likely to be macrosomic and more likely to be of low birth weight compared to the neonates of obese Caucasian women [31]. Our results showed that women of color were more often overweight or obese when compared with white women; however, a difference in cesarean rates was only noted between black women and other races. This suggests that there is an etiology beyond the contribution of obesity. There is also more recent literature in response to the 2009 Institute of Medicine guidelines for weight gain in pregnancy and the role of prepregnancy BMI, delivery BMI, weight gain in pregnancy, and even changes in BMI throughout pregnancy [37, 38]. It is evident that these are all potential variables that influence outcomes though the weight of their impact is not completely known. Black women were delivered for nonreassuring fetal heart rate tracing (NRFHRT) more often in comparison to other groups in our study, a finding that parallels the higher rate of fetal growth restriction in black women. It is plausible that black women have a predisposition to differences in placentation or antepartum uteroplacental flow that leads to a higher rate of fetal growth restriction. This chronic fetal nutritive and respiratory deprivation may predispose these fetuses to NRFHRT and thus cesarean delivery suggesting the root cause of the disparities demonstrated were factors that play well before entry into labor and perhaps well before conception. This study also occurred prior to current guidelines regarding categorization of fetal heart rate tracings [39, 40]. Although it was unable to be examined fully in this study, there may be bias in how these fetal tracings are differentially interpreted among the physicians and staff caring for these women. Fetal heart rate tracing interpretation is also fraught with both interobserver and intraobserver variation, so this may not ever be adequately explored. It is most likely that these differences result from multiple causes including biologic makeup of the individual as well as external influences such as provider bias and other practice differences.

Other quality measures including rates of postpartum hemorrhage and puerperal infection have been found to be higher in certain races. Previous studies show Hispanic women to have higher rates of postpartum hemorrhage when compared to other races $[41,42]$. Our findings were consistent with this and showed a 3.7 -fold and a 2.4 -fold increased risk of postpartum hemorrhage in Hispanic women compared to whites and blacks, respectively. Puerperal infection rates have also been shown to be higher among women of color compared to white women [5, 41]; however, we only found an increased rate of chorioamnionitis and endometritis in Hispanic women relative to whites and no increased risk for blacks. The higher rate of postpartum hemorrhage could be in part related to the higher rate of chorioamnionitis, a known risk factor for postpartum hemorrhage but would not be adequate to explain the significant difference noted. There was no difference in length of active labor or time to delivery between the groups. However, there were more Hispanic women induced for ruptured membranes, as well as oligohydramnios (almost twice the rate as for white women), and it is conceivable that this oligohydramnios reflects an even greater proportion of women with ruptured membranes, a known risk factor for chorioamnionitis. It is unclear what the etiology of these differences is as puerperal infections would be expected to be higher in black women given the higher rate of cesarean delivery which is a risk factor for both hemorrhage and infection. On the contrary, we found that both puerperal infection and postpartum hemorrhage were more common in Hispanic women despite similarities in length of labor among the races. Again, this suggests an etiology beyond known contributors and may be an inherent racial/ethnic difference that should help modify obstetric care. Nonetheless, duration of maternal stay was not significantly affected by this higher rate of postpartum hemorrhage and infection nor was neonatal outcomes significantly different for Hispanics compared to other race/ethnicity groups.

A limitation of this study is that aspects of acculturation and general socioeconomic statuses were not included. Other limitations include variable obstetric practices in this large, multicenter study, unknown baseline cesarean rates at specific hospitals, and breakdowns of site-specific influences that were not taken into account due to an inability to incorporate these factors in a practicable manner. The absence of standardized definitions for induction indications across all 49 centers presented an issue with regard to postdates induction as well 
as impending macrosomia as the subjects were enrolled and the data collected prior to most hospitals adopting more stringent criteria for inducing women. However, patients have been recategorized in compliance with ACOG recommendations as described in Practice Bulletin 107[43]. The main limitation of this study, however, is that it is a secondary analysis that was not powered specifically for differences in outcomes based on race/ethnicity but rather to look at outcomes for the different cervical ripening treatment groups. Though differences among racial/ethnic groups were not the primary outcomes of the initial study, multiple outcome variables were collected and presented a unique opportunity to evaluate the outcomes assessed in the secondary analysis.

A strength of this report is the incorporation of confounding factors such as body mass indices and morbidities associated with obesity into the secondary analyses. These factors affect the US population as a whole and often disproportionately affect black and Hispanic populations [5, 31, 32, 34, 35, 44, 45]. The races in this analysis were compared to each other as well as well as to whites as a reference group, and results emphasize the existence of interracial differences. Another asset of this evaluation is that the data were collected from 49 diverse sites across the USA and Canada during one of the largest prospective, double-blinded trials on prostaglandin induction using a standardized protocol increasing external validity and minimizing provider bias that has often been cited for differences in medical care. The original study was a prospective randomized controlled trial in which women who presented after 36 weeks 0 days that required cervical ripening were approached for participation thus eliminating selection bias and controlling for known and unknown confounding factors.

The literature is replete with reports of significant medical and obstetrical disparities based on race and ethnicity. These disparate outcomes and differences in health care challenge practitioners to address these disparities and attempt to identify possible practice strategies to better care for a diverse patient population. The causes of these disparities are largely unknown and are often multifactorial. It is apparent from previous literature that some of these factors may be modifiable but that many will be predetermined and may be helpful as risk counseling points. This report attempts to highlight persistence of disparities despite controlling for confounders and offer further avenues of research to better characterize differences between races. Given the multiple modifiers of antenatal, intrapartum, and postpartum health, it would be impossible to offer an exclusive cause for these disparities, and research should be focused on discovery of those disparities that may be amenable to change. Knowledge of these inherent differences between groups could help to identify a particular patient's risk factors, aid in provision of better health care, provide a safer delivery environment, and ultimately improve maternal and fetal outcomes of women affected by race-related morbidity.
Acknowledgments This research was funded by Controlled Therapeutics (Scotland) Ltd, wholly owned subsidiary of Cytokine PharmaSciences, Inc. In 2012, Ferring Pharmaceuticals, Inc. acquired Cytokine PharmaSciences, Inc. All procedures followed were in accordance with the ethical standards of the responsible committee on human experimentation (institutional and national) and with the Helsinki Declaration of 1975, as revised in 2000 [4]. Informed consent was obtained from all patients for being included in the study.

Conflict of Interest Drs. Stephenson, Hawkins, and Pevzner declare that they have no conflict of interest.

Dr. Powers is a former employee of Cytokine PharmaSciences, Inc.

Dr. Wing was a principal investigator for the sponsor and is a consultant for Ferring Pharmaceuticals.

\section{References}

1. Bryant AS, Washington S, Kuppermann M, Cheng YW, Caughey $\mathrm{AB}$. Quality and equality in obstetric care: racial and ethnic differences in caesarean section delivery rates. Paediatr Perinat Epidemiol. 2009;23(5):454-62.

2. Bryant AS, Worjoloh A, Caughey AB, Washington AE. Racial/ ethnic disparities in obstetric outcomes and care: prevalence and determinants. Am J Obstet Gynecol. 2010;202(4):335-43.

3. Centers for Disease C, Prevention. State-specific trends in U.S. live births to women born outside the 50 states and the District of Columbia-United States, 1990 and 2000. MMWR Morb Mortal Wkly Rep. 2002;51(48):1091-5.

4. Khalil A, Rezende J, Akolekar R, Syngelaki A, Nicolaides KH. Maternal racial origin and adverse pregnancy outcome: a cohort study. Ultrasound Obstet Gynecol: Off J Int Soc Ultrasound Obstet Gynecol. 2013;41(3):278-85.

5. Shen JJ, Tymkow C, MacMullen N. Disparities in maternal outcomes among four ethnic populations. Ethn Dis. 2005;15(3):492-7.

6. Chung JH, Garite TJ, Kirk AM, Hollard AL, Wing DA, Lagrew DC. Intrinsic racial differences in the risk of cesarean delivery are not explained by differences in caregivers or hospital site of delivery. Am J Obstet Gynecol. 2006;194(5):1323-8.

7. Le Cook B, McGuire TG, Zuvekas SH. Measuring trends in racial/ ethnic health care disparities. Med Care Res Rev: MCRR. 2009;66(1):23-48.

8. Alexander GR, Wingate MS, Bader D, Kogan MD. The increasing racial disparity in infant mortality rates: composition and contributors to recent US trends. Am J Obstet Gynecol. 2008;198(1):51.e1-9.

9. Healy AJ, Malone FD, Sullivan LM, Porter TF, Luthy DA, Comstock $\mathrm{CH}$, et al. Early access to prenatal care: implications for racial disparity in perinatal mortality. Obstet Gynecol. 2006;107(3):625-31.

10. Wing DA. Misoprostol Vaginal Insert C, Misoprostol vaginal insert compared with dinoprostone vaginal insert: a randomized controlled trial. Obstet Gynecol. 2008;112(4):801-12.

11. ACOG Committee Opinion No 579. Definition of term pregnancy. Obstet Gynecol. 2013;122(5):1139-40.

12. American College of Gynecologists. ACOG committee opinion no. 561. Nonmedically indicated early-term deliveries. Obstet Gynecol. 2013;121(4):911-5.

13. Spong CY. Defining "term" pregnancy: recommendations from the defining "term" pregnancy workgroup. JAMA: J Am Med Assoc. 2013;309(23):2445-6.

14. Stein M, O'Malley K, Kilfeather S. Ethnic differences in cyclic AMP accumulation: effect on alpha 2, beta 2 , and prostanoid receptor responses. Clin Pharmacol Ther. 1990;47(3):360-5. 
15. Gauthier T, Mazeau S, Dalmay F, Eyraud JL, Catalan C, Marin B, et al. Obesity and cervical ripening failure risk. J Matern-Fetal Neonatal Med: Off J Eur Assoc Perinatal Med, Fed Asia Ocean Perinatal Soc, Int Soc Perinatal Obstet. 2012;25(3):304-7.

16. Pevzner L, Powers BL, Rayburn WF, Rumney P, Wing DA. Effects of maternal obesity on duration and outcomes of prostaglandin cervical ripening and labor induction. Obstet Gynecol. 2009;114(6): 1315-21.

17. Martinez ME, Heddens D, Earnest DL, Bogert CL, Roe D, Einspahr $\mathrm{J}$, et al. Physical activity, body mass index, and prostaglandin E2 levels in rectal mucosa. J Natl Cancer Inst. 1999;91(11):950-3.

18. Menon R, Merialdi M, Lombardi SJ, Fortunato SJ. Differences in the placental membrane cytokine response: a possible explanation for the racial disparity in preterm birth. Am J Reprod Immunol. 2006;56(2): $112-8$.

19. Menon R, Williams SM, Fortunato SJ. Amniotic fluid interleukin1 beta and interleukin- 8 concentrations: racial disparity in preterm birth. Reprod Sci. 2007;14(3):253-9.

20. Martin JA, Hamilton BE, Ventura SJ, Osterman MJ, Kirmeyer S, Mathews TJ, et al. Births: final data for 2009. National Vital statistics reports: from the Centers for Disease Control and Prevention, National Center for Health Statistics. Nat Vital Stat Syst. 2011;60(1):1-70.

21. Kramer MS, Ananth CV, Platt RW, Joseph KS. US Black vs White disparities in foetal growth: physiological or pathological? Int $\mathrm{J}$ Epidemiol. 2006;35(5):1187-95.

22. Centers for Disease C, Prevention. Rates of cesarean delivery among Puerto Rican women-Puerto Rico and the U.S. mainland, 19922002. MMWR Morb Mortal Wkly Rep. 2006;55(3):68-71.

23. Brown HL, Chireau MV, Jallah Y, Howard D. The "Hispanic paradox": an investigation of racial disparity in pregnancy outcomes at a tertiary care medical center. Am J Obstet Gynecol. 2007;197(2): 197.e1-7. discussion e7-9.

24. Bennett TA, Adams MM. Safe motherhood in the United States: challenges for surveillance. Matern Child Health J. 2002;6(4):221-6.

25. Ruiz RJ, Dolbier CL, Fleschler R. The relationships among acculturation, biobehavioral risk, stress, corticotropin-releasing hormone, and poor birth outcomes in Hispanic women. Ethn Dis. 2006;16(4):926-32.

26. Kabir AA, Pridjian G, Steinmann WC, Herrera EA, Khan MM. Racial differences in cesareans: an analysis of U.S. 2001 National Inpatient Sample Data. Obstet Gynecol. 2005;105(4):710-8.

27. Braveman P, Egerter S, Edmonston F, Verdon M. Racial/ethnic differences in the likelihood of cesarean delivery, California. Am J Public Health. 1995;85(5):625-30.

28. Edmonds JK, Yehezkel R, Liao X, Moore Simas TA. Racial and ethnic differences in primary, unscheduled cesarean deliveries among low-risk primiparous women at an academic medical center: a retrospective cohort study. BMC Pregnancy Childbirth. 2013;13:168.

29. Getahun D, Strickland D, Lawrence JM, Fassett MJ, Koebnick C, Jacobsen SJ. Racial and ethnic disparities in the trends in primary cesarean delivery based on indications. Am J Obstet Gynecol. 2009;201(4):422.e1-7.
30. Ruiz RJ, Saade GR, Brown CE, Nelson-Becker C, Tan A, Bishop S, et al. The effect of acculturation on progesterone/estriol ratios and preterm birth in Hispanics. Obstet Gynecol. 2008;111(2 Pt 1):309-16.

31. Marshall NE, Guild C, Cheng YW, Caughey AB, Halloran DR. Racial disparities in pregnancy outcomes in obese women. $\mathrm{J}$ Matern-Fetal Neonat Med: Off J Eur Assoc Perinat Med Fed Asia Ocean Perinat Soc Int Soc Perinat Obstet. 2014;27(2):122-6.

32. Rosenberg TJ, Garbers S, Lipkind H, Chiasson MA. Maternal obesity and diabetes as risk factors for adverse pregnancy outcomes: differences among $4 \mathrm{racial} /$ ethnic groups. Am J Public Health. 2005;95(9): $1545-51$.

33. Swank ML, Caughey AB, Farinelli CK, Main EK, Melsop KA, Gilbert WM, et al. The impact of change in pregnancy body mass index on the development of gestational hypertensive disorders. J Perinatol. 2014;34:181-185

34. Pevzner L, Rayburn WF, Rumney P, Wing DA. Factors predicting successful labor induction with dinoprostone and misoprostol vaginal inserts. Obstet Gynecol. 2009;114(2 Pt 1):261-7.

35. Ramos GA, Caughey AB. The interrelationship between ethnicity and obesity on obstetric outcomes. Am J Obstet Gynecol. 2005;193(3 Pt 2):1089-93.

36. Steinfeld JD, Valentine S, Lerer T, Ingardia CJ, Wax JR, Curry SL. Obesity-related complications of pregnancy vary by race. J MaternFetal Med. 2000;9(4):238-41.

37. In: Rasmussen KM, Yaktine AL, editors. Weight gain during pregnancy: reexamining the guidelines. The National Academies Collection: Reports funded by National Institutes of Health. Washington (DC) 2009.

38. American College of Gynecologists. ACOG Committee opinion no. 548. Weight gain during pregnancy. Obstet Gynecol. 2013;121(1): $210-2$.

39. American College of Gynecologists. Practice bulletin no. 116: management of intrapartum fetal heart rate tracings. Obstet Gynecol. 2010;116(5):1232-40.

40. American College of O, Gynecologists. ACOG Practice Bulletin No. 106. Intrapartum fetal heart rate monitoring: nomenclature, interpretation, and general management principles. Obstet Gynecol. 2009;114(1):192-202.

41. Guendelman S, Thornton D, Gould J, Hosang N. Obstetric complications during labor and delivery: assessing ethnic differences in California. Women's Health Issues. 2006;16(4):189-97.

42. Combs CA, Murphy EL, Laros Jr RK. Factors associated with postpartum hemorrhage with vaginal birth. Obstet Gynecol. 1991;77(1):69-76.

43. Obstetrics ACoPB-. ACOG Practice Bulletin No. 107. Induction of labor. Obstet Gynecol. 2009;114(2 Pt 1):386-97.

44. Rosenberg TJ, Garbers S, Chavkin W, Chiasson MA. Prepregnancy weight and adverse perinatal outcomes in an ethnically diverse population. Obstet Gynecol. 2003;102(5 Pt 1):1022-7.

45. Flegal KM, Carroll MD, Kit BK, Ogden CL. Prevalence of obesity and trends in the distribution of body mass index among US adults, 1999-2010. JAMA: J Am Med Assoc. 2012;307(5):491-7. 